\title{
Characteristics of recurrence after radical esophagectomy with two-field lymph node dissection for thoracic esophageal cancer
}

\author{
CHENG-LIN LI ${ }^{1,2^{*}}$, FU-LI ZHANG ${ }^{1 *}$, YA-DI WANG ${ }^{1}$, CHUN HAN $^{2}$, GUO-GUI SUN ${ }^{3}$, \\ QING LIU ${ }^{2}$, YUN-JIE CHENG ${ }^{2}$, SHAO-WU JING $^{2}$ and CONG-RONG YANG ${ }^{2}$ \\ ${ }^{1}$ Department of Radiation Oncology, Military General Hospital of Beijing PLA, Beijing 100700; \\ ${ }^{2}$ Department of Radiation Oncology, Fourth Affiliated Hospital of Hebei Medical University, Shijiazhuang 050011; \\ ${ }^{3}$ Department of Radiation Oncology, The People's Hospital of Tangshan, Tangshan 063001, P.R. China
}

Received May 17, 2012; Accepted September 11, 2012

DOI: $10.3892 / \mathrm{ol} .2012 .946$

\begin{abstract}
Postoperative radiotherapy has shown positive efficacy in lowering the recurrence rate and improving the survival rate in cases involving lymph node (LN) metastasis. However, the radiotherapy target volume remains controversial. Certain published studies have paid more attention to LNs found to be affected during surgery, while little effort has been made to study the LN metastatic pattern following surgery and its influence on the determination of the target volume of postoperative radiotherapy. In this study, the locoregional recurrence of esophageal squamous cell cancer was examined in 134 patients receiving radical surgery with two-field lymph node dissection from 2004 to 2009. In the 134 cases of recurrence, LN metastasis occurred in 126 patients (94.0\%) while 13 patients (9.7\%) developed anastomotic recurrence and 5 patients $(3.7 \%)$ experienced tumor bed recurrence. The difference among the groups was statistically significant $(\mathrm{P}=0.000)$. In the 126 cases with lymph node metastasis, the mediastinal metastasis rate $(80.2 \%)$ was significantly higher compared with the rate of supraclavicular metastasis and abdominal metastasis $(\mathrm{P}=0.000)$. A significant difference was identified between right and left supraclavicular $\mathrm{LN}$ metastasis $(31.7 \%$ vs $16.7 \%, \mathrm{P}=0.005)$. Furthermore, the difference between the metastatic rates in the upper $(73.8 \%)$, middle (39.7\%) and lower mediastinum $(1.6 \%)$ was statistically significant $(\mathrm{P}=0.000)$. Nevertheless, no significant correlation between the rate of LN metastasis was observed in the supraclavicular, mediastinal and abdominal regions for upper, middle and lower thoracic
\end{abstract}

Correspondence to: Professor Ya-Di Wang, Department of Radiation Oncology, Military General Hospital of Beijing PLA, 5 Nanmencang, Dongcheng, Beijing 100700, P.R. China

E-mail: wangyadi@hotmail.com

${ }^{*}$ Contributed equally

Key words: esophageal squamous cell carcinoma, lymph node metastasis, postoperative recurrence, two-field esophagectomy carcinomas $(\mathrm{P}=0.404, \mathrm{P}=0.718$ and $\mathrm{P}=0.169$, respectively). Based on our data, LN metastasis is the major locoregional recurrence pattern for esophageal squamous cell cancer following radical surgery. The high-risk lymphatic drainage areas include the supraclavicular nodes, recurrent laryngeal nerve nodes, azygos nodes and subcarinal nodes.

\section{Introduction}

Surgery is the mainstay treatment for resectable esophageal cancer. Although the long-term survival rate associated with esophageal cancer remains at approximately $15 \%$ worldwide (1), certain recent studies have described an increase in the overall 5-year survival rate of $40 \%$ or more following esophagectomy with three-field lymphadenectomy (2), but with more complications. Postoperative radiotherapy has shown positive efficacy in lowering the recurrence rate and improving the survival rate in cases involving lymph node (LN) metastasis (3-5), although it remains unknown whether postoperative radiotherapy improves survival in all cases $(3,6)$. Reasons for this controversy may be the undetermined target volume or insensitivity of the tissue to radiotherapy due to ischemia and hypoxia following surgery. A large T-shaped field was first used which encompassed the bilateral supraclavicular fossi, mediastinum and left gastric nodes. In recent years, the large $\mathrm{T}$-shaped field was modified into a smaller T-shaped field to reduce unacceptable complication rates by omitting the left gastric artery drainage region (5). Certain published studies have focussed on LNs found to be affected during surgery (7-9) while little work has been done to study the LN metastasis pattern following surgery and its influence on the determination of the target volume of postoperative radiotherapy. To our knowledge, to date, only Cai et al (10) have studied the pattern of relapse in patients who received esophagectomy with two-field or three-field lymphadenectomy for thoracic esophageal cancer and provided some reference for the determination of the postoperative radiotherapy target volume for esophageal cancer. Based on this study, we further analyzed the locoregional recurrence patterns following radical esophagectomy focussing on two-field LN dissection for thoracic esophageal cancer. 
Table I. Correlation between tumor location and $\mathrm{T}$ and $\mathrm{N}$ stages.

\begin{tabular}{|c|c|c|c|c|c|c|c|c|c|}
\hline \multirow[b]{2}{*}{ Tumor location } & \multirow[b]{2}{*}{ No. of cases } & \multicolumn{4}{|c|}{ T stage } & \multicolumn{4}{|c|}{$\mathrm{N}$ stage } \\
\hline & & $\mathrm{T} 1$ & $\mathrm{~T} 2$ & $\mathrm{~T} 3$ & $\mathrm{~T} 4$ & No & $\mathrm{N} 1$ & $\mathrm{~N} 2$ & N3 \\
\hline Upper TEC & 17 & 4 & 4 & 8 & 1 & 11 & 5 & 1 & 0 \\
\hline Middle TEC & 99 & 9 & 16 & 66 & 8 & 55 & 31 & 12 & 1 \\
\hline Lower TEC & 18 & 1 & 3 & 10 & 4 & 12 & 5 & 1 & 0 \\
\hline Total & 134 & 14 & 23 & 84 & 13 & 78 & 41 & 14 & 1 \\
\hline
\end{tabular}

TEC, thoracic esophageal cancer.

Table II. Recurrence type classification.

\begin{tabular}{lccc}
\hline Recurrence type & No. of cases (\%) & $\chi^{2}$ value & P-value \\
\hline Metastasis of the LN & $126(94.0)$ & 297.280 & 0.000 \\
Recurrence of anastomosis & $13(9.7)$ & & \\
Recurrence of primary tumor & $5(3.7)$ & & \\
\hline
\end{tabular}

LN, lymph node.

\section{Materials and methods}

General information. The clinical data of 134 patients treated in our department from 2004 to 2009 for recurrent thoracic esophageal squamous cell carcinoma following radical surgery were collected. These included 101 male patients and 33 female patients. Seventeen cases had upper thoracic esophageal cancer, 99 had middle thoracic esophageal cancer, and 18 had lower thoracic esophageal cancer. The patients were staged postoperatively according to the UICC TNM staging system (11), as shown in Table I. Radical esophagectomy with two-field lymphadenectomy was performed on all patients and no patient received prophylactic radiotherapy following surgery. The time from the end of the surgery to recurrence ranged from 0.5 to 96 months, with a median recurrence time of 12 months. The study was approved by the Ethics Committee of the Military General Hospital of Beijing PLA, Beijing, China. Written informed patient consent was obtained from the patient.

Diagnosis standard. Locoregional recurrence patterns were classified into three types based on recurrence at the site of the primary tumor, the anastomotic site or metastasis of the LN. LN metastasis is defined as recurrent disease at LNs in the cervical, mediastinal and abdominal areas with a single $\mathrm{LN} \geq 1 \mathrm{~cm}$ in the smallest dimension, $\mathrm{LN} \geq 3 \mathrm{~cm}$ at a single site or a single paratracheal $\mathrm{LN}<1 \mathrm{~cm}$ in the smallest dimension combined with hoarseness or vocal cord paralysis. Recurrence was diagnosed based on histopathological or radiological (on follow-up CT or PET/CT) evidence.

Boundary demarcation. Boundaries of $\mathrm{LN}$ regions were according to the CT-based definition of anatomical landmarks (12). Stations 1-2 comprise the highest mediastinal and upper paratracheal nodes; Station 3, prevascular nodes and retrotracheal nodes; Station 4, lower paratracheal nodes; Station 5, subaortic nodes; Station 6, paraaortic nodes or anterior aortic nodes; Station 7, subcarinal nodes; Station 8, paraesophageal nodes and Station 10 comprises the hilar nodes. It should be noted that Station 9, comprising pulmonary ligament nodes, was not delineated since its location is defined in part by the inferior pulmonary ligament, which is typically not visible on CT images (12).

Stations 1, 2 and 4 were separated by the midline of the trachea into $1 \mathrm{R}$ and $1 \mathrm{~L}, 2 \mathrm{R}$ and $2 \mathrm{~L}$, and $4 \mathrm{R}$ and $4 \mathrm{~L}$, respectively. Station 8 was separated by the end of the left inferior pulmonary artery into 8S and 8I. Stations 1, 2, 3, 4, 5 and 6 were located in the upper mediastinum and Stations 7, 10 and the upper part of Station 8 were located in the middle mediastinum while Station 9 and the lower part of Station 8 were located in the lower mediastinum.

Calculation of the recurrence and metastatic rate of LNs. The recurrence and LN metastasis rates were calculated as follows: recurrence rate $=$ number of cases of recurrence/total number of cases $\mathrm{x} 100 \%$; LN metastasis rate $=$ number of cases of metastasis / total number of cases x $100 \%$.

Statistical analysis. Statistical analysis was performed using SPSS software (version 18.0, SPSS Inc., Chicago, IL, USA). A $\chi^{2}$ test was used for enumeration data. A value of $\mathrm{P} \leq 0.05$ was considered to indicate a statistically significant result.

\section{Results}

Recurrence types. The recurrence types are classified in Table II. LN metastasis was the most common type of recur- 
Table III. Correlation between LN site and corresponding metastatic rates.

\begin{tabular}{|c|c|c|c|c|}
\hline Location of LN & No. of cases & Metastatic rate $(\%)$ & $\chi^{2}$ value & P-value \\
\hline Supraclavicular & 55 & 43.7 & $113.150^{\mathrm{a}}$ & $0.000^{\mathrm{a}}$ \\
\hline Left supraclavicular & 21 & 16.7 & $7.808^{\mathrm{b}}$ & $0.005^{b}$ \\
\hline Right supraclavicular & 40 & 31.7 & & \\
\hline Mediastinal & 101 & 80.2 & $139.117^{c}$ & $0.000^{\mathrm{c}}$ \\
\hline Upper mediastinal & 93 & 73.8 & $130.029^{\mathrm{d}}$ & $0.000^{\mathrm{d}}$ \\
\hline $1 \mathrm{R}$ & 55 & 43.7 & & \\
\hline $2 \mathrm{R}$ & 32 & 25.4 & & \\
\hline $4 \mathrm{R}$ & 27 & 21.4 & & \\
\hline $1 \mathrm{~L}$ & 19 & 15.1 & & \\
\hline $2 \mathrm{~L}$ & 18 & 14.3 & & \\
\hline $4 \mathrm{~L}$ & 19 & 15.1 & & \\
\hline 5 & 16 & 12.7 & & \\
\hline $3 \mathrm{~A}$ & 5 & 4.0 & & \\
\hline $3 \mathrm{P}$ & 5 & 4.0 & & \\
\hline 6 & 3 & 2.4 & & \\
\hline Middle mediastinal & 50 & 39.7 & $82.064^{\mathrm{e}}$ & $0.000^{\mathrm{e}}$ \\
\hline 7 & 43 & 34.1 & & \\
\hline $8 \mathrm{~S}$ & 5 & 4.0 & & \\
\hline $10 \mathrm{R}$ & 4 & 3.2 & & \\
\hline $10 \mathrm{~L}$ & 7 & 5.6 & & \\
\hline Lower mediastinal & 2 & 1.6 & & \\
\hline $8 \mathrm{I}$ & 1 & 0.8 & & \\
\hline 9 & 1 & 0.8 & & \\
\hline Abdominal & 17 & 13.5 & & \\
\hline
\end{tabular}

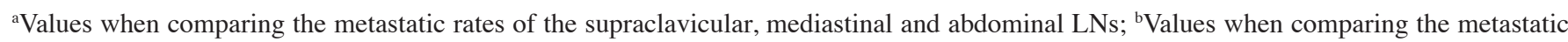
rates of the left and right supraclavicular LNs; 'Values when comparing the metastatic rates of the upper, middle and lower mediastinal

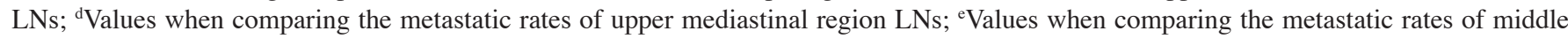
mediastinal region LNs. LN, lymph node.

rence in our study, occurring in $94 \%$ of cases. The recurrence rates of the anastomotic site and the primary tumor were $9.7 \%$ and $3.7 \%$, respectively. The differences between the three groups were statistically significant $(\mathrm{p}=0.000)$.

Location of LN metastasis. As shown in Table III, mediastinal LNs had the highest metastatic rates, reaching $80.2 \%$, followed by supraclavicular LNs $(43.7 \%)$, while the metastatic rate of abdominal LNs was $13.5 \%$. The differences between the three groups were statistically significant $(\mathrm{P}=0.000)$. Twenty-one patients suffered from left supraclavicular LN metastasis, accounting for $38.2 \%$ of patients having supraclavicular LN metastasis and $16.7 \%$ of total patients with LN metastasis. Right supraclavicular LN metastasis occurred in 40 patients, accounting for $72.7 \%$ of patients suffering from supraclavicular metastasis and $31.7 \%$ of total patients with LN metastasis. The number of patients with right supraclavicular LN metastasis was larger than that of patients experiencing left supraclavicular metastasis, with a statistically significant difference $(\mathrm{P}=0.005)$. The upper mediastinum was the site where most mediastinal LN metastasis occurred, accounting for $73.8 \%$ of the total cases suffering from LN metastasis, followed by the middle $(39.7 \%)$ and lower mediastinum (1.6\%). The differences between the three groups were statistically significant $(\mathrm{P}=0.000)$.

The metastatic rates of the supraclavicular, mediastinal and abdominal LN showed no statistically significant difference for esophageal squamous cell carcinomas in the upper, middle and lower thoracic regions $(\mathrm{P}=0.404, \mathrm{P}=0.718$ and $\mathrm{P}=0.169$, respectively), as indicated in Table IV.

As demonstrated in Table III, LN metastasis of the upper mediastinum appeared in the following regions: 1R $(43.7 \%)$, 2R (25.4\%), 4R (21.4\%), 1L (15.1\%), 2L (14.3\%), 4L (15.1\%) and Station $5(12.7 \%)$. The differences among the groups were statistically significant $(\mathrm{P}=0.000)$. $\mathrm{LN}$ metastasis of the middle mediastinum mainly occurred in Station 7 (34.1\%) followed by $10 \mathrm{~L}(5.6 \%), 8 \mathrm{~S}(4.0 \%)$ and $10 \mathrm{R}(3.2 \%)$, with statistically significant differences $(\mathrm{P}=0.000)$. $\mathrm{LN}$ metastasis of the lower mediastinum occurred rarely and the metastatic rate of $8 \mathrm{I}$ plus Station 9 was $1.6 \%$. The metastatic rate of LNs along the left recurrent laryngeal nerve throughout their mediastinal course, including 1L, 2L, 4L and Station 5 was $38.9 \%$, while LNs along the right recurrent laryngeal nerve had a metastatic rate of $43.7 \%$. The difference between the two groups was not statistically significant, as demonstrated in Table V. 
Table IV. Relationship between primary tumor locations and LN metastasis rates.

\begin{tabular}{|c|c|c|c|c|c|c|}
\hline Tumor location & No. of cases & No. of SM (\%) & No. of MM (\%) & No. of AM (\%) & $\chi^{2}$ value & P-value \\
\hline Upper TEC & 16 & $9(56.3)$ & $12(75.0)$ & $1(6.3)$ & $1.813^{\mathrm{a}}$ & $0.404^{\mathrm{a}}$ \\
\hline Middle TEC & 92 & $40(43.5)$ & $75(81.5)$ & $11(12.0)$ & $0.710^{\mathrm{b}}$ & $0.718^{\mathrm{b}}$ \\
\hline Lower TEC & 18 & $6(33.3)$ & $14(77.8)$ & $5(27.8)$ & $3.548^{\mathrm{c}}$ & $0.169^{c}$ \\
\hline
\end{tabular}

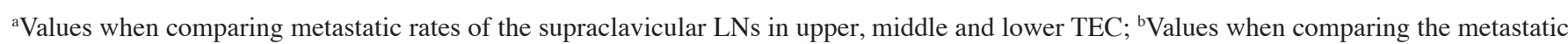
rates of the mediastinal LNs in upper, middle and lower TEC; 'Values when comparing the metastatic rates of abdominal LNs in upper, middle and lower TEC. LN, lymph node; TEC, thoracic esophageal cancer; SM, supraclavicular metastasis of LN; MM, mediastinal metastasis; $\mathrm{AM}$, abdominal metastasis.

Table V. Distribution of recurrent laryngeal nerve LN metastasis.

\begin{tabular}{lccc}
\hline Location & No. of cases & Metastatic rate (\%) & $\chi^{2}$ value \\
\hline $\begin{array}{l}\text { Left recurrent laryngeal nerve } \\
(1 \mathrm{~L}, \text { 2L, 4L, Station 5) }\end{array}$ & 49 & 38.9 & 0.589 \\
$\begin{array}{l}\text { Right recurrent laryngeal nerve } \\
(1 \mathrm{R})\end{array}$ & 55 & 43.7 & 0.443 \\
\hline
\end{tabular}

LN, lymph node.

\section{Discussion}

Currently, surgery is a mainstay of treatment for esophageal squamous cell cancer. However, the overall 5-year survival rate is not satisfactory. Locoregional recurrence is one of the important factors influencing prognosis following surgery. A study reported that $50 \%$ of early mortality cases were caused by locoregional recurrence rather than distant metastasis (13).

With the improvement of surgical approach and skill, the resection rate of thoracic esophageal cancer has increased to over $90 \%$ (14), while the local and anastomotic recurrence rates have significantly reduced. In our study, the recurrence rate of the anastomotic site was $9.7 \%$, which is comparable to the rates of $3-9 \%$ reported in previous reports (8). One of the reasons may be that the anastomotic positions of patients in the study of Lee et al were higher, which reduced the risk of anastomotic recurrence. Ninety-seven cases in our group were in advanced stage (T3 or T4) and suffering from deep invasion. Severe adhesion of the primary tumor to surrounding tissue was found in 49 cases at the time of surgery, which made the resection difficult; however, only 5 cases experienced recurrence of the tumor bed, accounting for $3.7 \%$ of the total. Furthermore, the recurrence of these 5 cases exclusively occurred in upper or middle thoracic esophageal cancer, which may be due to incomplete tumor exposure during surgery.

In this study, LN metastasis was the most common recurrence type of thoracic esophageal cancer with a rate of $94.0 \%$. Chen et al (15) reported a locoregional recurrence rate of $54.1 \%$ at 3 years, with mediastinal LN metastasis accounting for $78.8 \%$.

Further analysis indicated that the order from highest to lowest metastatic rate for the different $\mathrm{LN}$ regions was upper mediastinum (73.8\%), supraclavicular site (43.7\%), middle mediastinum (39.7\%), abdomen (13.5\%) and lower mediastinum (1.6\%). Cervical, middle and upper mediastinal LN had higher metastatic rates which correlate with the findings of Cai et al (10). In addition, the metastatic rates of $1 \mathrm{R}(43.7 \%)$ and Station 7 (34.1\%) were higher than those of $2 \mathrm{R}(25.4 \%)$ and $4 \mathrm{R}$ (21.4\%), while 1L (15.1\%), 2L (14.3\%), 4L (15.1\%) and Station 5 $(12.7 \%)$ had relatively low metastatic rates, which is consistent with the results of Liu et al (16).

Right upper mediastinal LN metastasis mainly appeared in $1 \mathrm{R}$ rather than $2 \mathrm{R}$ and $4 \mathrm{R}$, while the distribution of left upper mediastinal $\mathrm{LN}$ metastasis was fairly uniform $(1 \mathrm{~L}, 15.1 \%$; $2 \mathrm{~L}$, $14.3 \%$; 4L, 15.1\%; Station 5, 12.7\%). The right recurrent laryngeal nerve arises from the right vagus nerve and bypasses the right subclavian artery moving upwards along the $1 \mathrm{R}$ region. The left recurrent laryngeal nerve starts from the left vagus nerve and bypasses the aortic arch moving downwards along Station 5, 4L, 2L and 1L. As a result, metastatic LNs spread along two recurrent laryngeal nerves. The metastatic rates of $\mathrm{LN}$ along the left and right recurrent laryngeal nerve regions were similar (43.7 vs. $38.9 \%, \mathrm{P}=0.443$ ). Jang et al (17) reported $\mathrm{LN}$ metastatic rates in the right and left recurrent laryngeal nerve regions of patients undergoing lymphadenectomy for esophageal cancer through an incision of the right chest to be 32 and $26 \%$, respectively. These results were similar; however, they were inconsistent with the results of our study. A possible reason for this is the difference between the surgery-based LN station classification and CT-based LN station classification, leading us to assort the metastatic $\mathrm{LN}$ abutting right recurrent laryngeal nerve into the $2 \mathrm{R}$ region, resulting in a lower right recurrent laryngeal nerve LN metastatic rate. However, 19 out of 27 cases with LN metastasis of 4R not included in the recurrent laryngeal nerve region experienced azygos vein $\mathrm{LN}$ metastasis. Therefore, in thoracic esophageal 
cancer, upper mediastinal LNs mostly metastasize along the recurrent laryngeal nerve region. Incomplete $\mathrm{LN}$ dissection in this region, due to factors such as local complex anatomy and technical difficulty of dissection, may leave behind subclinical foci of disease.

According to the above analysis, we found that the LN region surrounding the recurrent laryngeal nerve and two other independent $\mathrm{LN}$ regions (the azygos vein site and subcarinal site) belonged to high risk locations where mediastinal LN metastasis occurred following radical esophagectomy for thoracic esophageal cancer. There are two possible reasons for this. Firstly, the occlusion of the aortic arch and right subclavian artery when performing lymphadenectomy through an incision of the left chest makes it difficult to dissect upper mediastinal LNs including those surrounding the recurrent laryngeal nerve. Secondly, the complex anatomy and the abundance of nerves and large blood vessels, especially the tight connection between the recurrent laryngeal nerve and surrounding LNs greatly increases the technical difficulty of surgery and may cause an omission of LNs during lymphadenectomy. By contrast, the lower mediastinum and upper abdominal sites can be well exposed and there are only certain paraesophageal and pulmonary ligament $\mathrm{LN}$ regions which have a relatively centralized distribution in the lower mediastinum, therefore the $\mathrm{LN}$ dissection is comparatively more thorough.

Recent studies regarding three-field lymphadenectomy have indicated that the cervical LN metastatic rates for upper, middle and lower thoracic esophageal cancer were 41.6-46.3\%, $29.2-33.3 \%$ and $27-36.4 \%$, respectively $(9,18)$. Patients in our study all underwent thoracic and abdominal two-field lymphadenectomy and the failure to remove potentially metastatic LNs in the supraclavicular site during surgery may be the cause of recurrence. It should be noted that the metastatic rate of right supraclavicular LNs $(31.7 \%)$ was significantly higher than that of left supraclavicular LNs (16.7\%), which corresponded to the phenomenon that the metastatic rate of $1 \mathrm{R}$ $(43.7 \%)$ was significantly higher than that of $1 \mathrm{~L}(15.1 \%)$. The correlation between these two cases is a topic of future studies. Certain researchers have noted that $\mathrm{LN}$ metastasis along recurrent laryngeal nerves is an independent prognostic factor for judging whether cervical LNs have metastasized (19).

In summary, LN metastasis was the most common type of recurrence for thoracic esophageal cancer in our study, followed by the recurrence of the primary tumor and the anastomotic site. The LNs in the supraclavicular, recurrent laryngeal nerves, azygos vein and subcarinal regions are high risk areas for locoregional metastasis, demonstrating that the target volume of the adjuvant radiotherapy following surgery should include supraclavicular and upper mediastinal LN regions, particularly recurrent laryngeal nerve LN sites. Whether the primary tumor bed and anastomosis require irradiation should be evaluated further in prospective clinical research.

\section{References}

1. Jemal A, Siegel R, Ward E, et al: Cancer statistics, 2008. CA Cancer J Clin 58: 71-96, 2008.

2. Tachimori Y and Kato H: Diagnosis and surgery of esophageal cancer. Crit Rev Oncol Hematol 28: 57-71, 1998.

3. Xiao ZF, Yang ZY, Miao YJ, et al: Influence of number of metastatic lymph nodes on survival of curative resected thoracic esophageal cancer patients and value of radiotherapy: report of 549 cases. Int J Radiat Oncol Biol Phys 62: 82-90, 2005.

4. Chen JQ, Zhu J, Pan JJ, et al: Postoperative radiotherapy improved survival of poor prognostic squamous cell carcinoma esophagus. Ann Thorac Surg 90: 435-442, 2010

5. Chen JQ, Pan JJ, Zheng XW, et al: Number and location of positive nodes, postoperative radiotherapy, and survival after esophagectomy with three-field lymph node dissection for thoracic esophageal squamous cell carcinoma. Int J Radiat Oncol Biol Phys: 2010, Epub ahead of print.

6. Ténière $\mathrm{P}$, Hay J-M, Fingerhut A, et al: Postoperation radiation therapy does not increase survival after curative resection for squamous cell carcinoma of the middle and lower esophagus as shown by a multicenter controlled trial. Surg Gynecol Obstet 173: 123-130, 1991.

7. Nakagawa S, Kanda T, Kosugi S, et al: Recurrence pattern of squamous cell carcinoma of the thoracic esophagus after extended radical esophagectomy with three-field lymphadenectomy. J Am Coll Surg 198: 205-211, 2004.

8. Lee SJ, Lee KS, Yim YJ, et al: Recurrence of squamous cell carcinoma of the oesophagus after curative surgery: rates and patterns on imaging studies correlated with tumour location and pathological stage. Clin Radiol 60: 547-554, 2005.

9. Li H, Zhang Y, Cai H, et al: Pattern of lymph node metastases in patients with squamous cell carcinoma of the thoracic esophagus who underwent three-field lymphadenectomy. Eur Surg Res 39: 1-6, 2007.

10. Cai WJ and Xin PL: Pattern of relapse in surgical treated patients with thoracic esophageal squamous cell carcinoma and its possible impact on target delineation for postoperative radiotherapy. Radiother Oncol 96: 104-107, 2010.

11. Sobin LH and Wittekind CH: TNM classification of malignant tumors. 6th edition. Wiley Liss, New York, 2002.

12. Chapet O, Kong FM, Quint LE, et al: CT-based definition of thoracic lymph node stations: an atlas from the University of Michigan. Int J Radiat Oncol Biol Phys 63: 170-178, 2005.

13. Giuli R and Gignoux M: Treatment of carcinoma of the esophagus. Retrospective study of 2,400 patients. Ann Surg 192: 44-52, 1980.

14. Ando N, Ozawa S, Kitagawa Y, et al: Improvement in the results of surgical treatment of advanced squamous esophageal carcinoma during 15 consecutive years. Ann Surg 232: 225-232, 2000.

15. Chen G, Wang Z, Liu XY, et al: Recurrence pattern of squamous cell carcinoma in the middle thoracic esophagus after modified Ivor-Lewis esophagectomy. World J Surg 31: 1107-1114, 2007.

16. Liu M, Li CY, Zhou DA, et al: The sites of lymph node metastasis in mediastinum after radical surgery for middle thoracic esophagus cancer. Chinese Journal of Radiation Oncology 2: 83-84, 2001.

17. Jang HJ, Lee HS, Kim MS, et al: Patterns of lymph node metastasis and survival for upper esophageal squamous cell carcinoma. Ann Thorac Surg 92: 1091-1098, 2011.

18. Akiyama $\mathrm{H}$, Tsunimaru $\mathrm{M}$, Udagawa $\mathrm{H}$, et al: Radical lymph node dissection for cancer of the thoracic esophagus. Ann Surg 2203: 364-372, 1994.

19. Shimada Y, Sato F, Maeda M, et al: Validity of intraoperative pathological diagnosis of paratracheal $\mathrm{LN}$ as a strategy forselection of patients for cervical lymph node dissection during egophagectomy. Dis Esophagus 16: 246-251, 2003. 